\title{
Querying Temporal Databases Using Controlled Natural Language*
}

\author{
Rani Nelken Nissim Francez \\ Computer Science Department \\ The Technion \\ Haifa 32000, Israel
}

\begin{abstract}
Recent years have shown a surge in interest in temporal database systems, which allow users to store time-dependent information. We present a novel controlled natural language interface to temporal databases, based on translating natural language questions into SQL/Temporal, a temporal database query language. The syntactic analysis is done using the Type-Logical Grammar framework, highlighting its utility not only as a theoretical framework but also as a practical tool. The scmantic analysis is done using a novel theory of the semantics of temporal questions, focusing on the role of temporal preposition phrases rather than the more traditional focus on tense and aspect. Our translation method is considerably simpler than previous attempts in this direction. We present a prototype software implementation.
\end{abstract}

\section{Introduction}

Traditionally, database management systems were designed to store snapshot information, valid at a particular moment of time (state). However, many applications require handling dynamic time-dependent information, pertaining not only to the present, but also to the past and future. Adding temporal support to databases has proved to be a surprisingly

\footnotetext{
* This work was carried out as part of the research project "Scmantics of Natural Language Temporal Questions and Interfaces to Temporal Database Systems" sponsored by the Fund for interdisciplinary research, administcred by the Isracli Academy of Science. We thank Michael Böhlen, Bob Carpenter and Andreas Steiner for each allowing us to incorporate their software within our own. We also thank Yoad Winter and the anonymous referees for helpful comments on a previous version of this paper. The work of the second author was partially supported by the fund for the promotion of research in the Technion.
}

thorny issue (Tansel et al., 1993). A recent drive to consolidate research efforts has led to the design of a consensus temporal data model and associated tomporal database query language, SQL/Temporal, an extension of the popular Structured Query Language (SQL) (Snodgrass, 2000). ${ }^{1}$ SQL/Temporal represents a significant improvement over standard $\mathrm{SQL}$ in allowing programmers to express temporal queries (Snodgrass, 2000). Since temporal database (TDB) implementations are still in their infancy (Boehlen, 1995), there is litthe practical experience with SQL/Temporal; let alone experience of non-expert users. However, it is our belief that such users are bound to find the expression of complex temporal queries in SQL/T'Temporal to be extremely difficult.

In an attempt to counter this problem, we present a translation method from controlled natural language (NL) to SQL/Temporal. Following the standard pipeline architecture of such methods, translation is done via an intermediate meaning representation language illustrated in Figure 1. NL questions are first parsed using a grammar in the Type Logical Grammar (TLG) framework (Carpenter, 1998; Morrill, 1998). Simultaneously with parsing, the NL question is translated into a formula of a formal language called $\mathrm{L}_{\text {Allen }}$ (Toman, 1996), based on the interval operators of (Allen, 1983). The translation is based on an independently motivated novel semantics of sentences modified by temporal Preposition Plhases (PPs) (Pratt and Francez 1997; 2000, Nelken and Francez 1999). The constructed $\mathrm{L}_{\text {Allen }}$ formula is then trans-

\footnotetext{
${ }^{1}$ Through continued design, SQL/Temporal has evolved from predecessor versions named TSQL2 (Snodgrass, 1995) and ATSQL2 (Boehlen et al., 1996). It is expected to be incorporated within the new version of SQL named SQL3.
} 
lated into an SQI./Temporal query, which is subsequently submitted to a prototype TDBB implementation for evaluation. Finally, the TIJB's answer is presented to the user.

$$
\mathrm{NL}, \rightarrow \mathrm{L}_{\text {Allen }} \rightarrow \mathrm{SQL}_{\mathrm{S} / \text { I'emporal }} \rightarrow \mathrm{TDB}
$$

Figure 1: The translation pipeline

We have implemented this method as a prototype software tool, called QWERT'Y, (almost) an acronym for "Querying with English of Relational Temporal Dalabases". P'arsing and translation to $\mathrm{L}_{\mathrm{A} \text { llen }}$ is done using the TLG Theorem Prover (Carpenter, 1999). The translation from $\mathrm{L}_{\mathrm{All}}$ to to $\mathrm{SQL} /$ /Temporal is done using an adaptation of a temporal logic (TT) to ATSQL2 translator of (Bochlen et al., 1996). 'The resulting query is submitted to a prototype TIDB implementation, called Timel)l3 (Steiner, 1997). The different modules are coupled into an integxated system implemented in Sicstus P'rolog on a UNIX platform with a WWW-based graphical front-end. We discuss some of the directions required in order to turn the system from a research prototype to a working tool.

\section{Related work}

There is voluminous literature on the design of $\mathrm{NI}$ interfaces to general (non-temporal) databases (sec (Permalt and Gros\% 1988; Copestake and Jones, $1990 ;$ Anclroutsopoulos et al., 1995) for surveys) and by now their main advantages and disadvantages are well understood. Much less work has been devoted to the design of NL, interfaces to TDBs (Clifford, 1990; Ilinrichs, 1988) or other computer systems involving a temporal dimension (Crouch and l'ulman, 1993). Of particular relevance is ( $\Lambda$ ndroutsopoulos, 1996), who presents a linguistically motivated translation method from NL queries into 'TSQL2 using an HPSG (Pollard and Sag, 1994) grammar and a TL as an intermediate representation language. Our approach shares many characteristics with (Androutsopoulos, 1996), but there also important differences, which we point out throughout the paper.

We begin our presentation of the translation method with a brief overview of the 'I'J) structure determines many of the deign choices taken in devising the translation method.

\section{The TDB}

A TIDB is a two-sorted first-order structure. The domain consists of a Dala Domain, D, and a Temporal Domain of intervals, $T_{I}$, defined as follows. Let $T_{P}$ be a set (of time points) with a discrete linear order without endpoints, $\leq . T_{I}$ is defined as the set of pairs : $T_{I}=$ $\{\langle a, b\rangle \mid a \leq b \wedge a, b \in T \cup\{-\infty, \infty\}\}$. A relational database schema is a set of single-sorted predicate symbols $\left(R_{1}, \ldots, R_{k}\right)$. Given a relational database schema $\rho$, a TDB schema $\rho^{\prime}$ is the set of two-sorted predicate symbols $\left(R_{1}^{\prime}, \ldots, R_{k}^{\prime}\right)$, where the sort of $R_{i}^{\prime}$ is $D^{\text {arity }(}\left(R_{i}\right) \times T_{I}$. A TIDB instance of schema $\rho^{\prime}$ is a set of rolations $\mathbf{R}_{\mathrm{i}}^{\prime} \subseteq D^{\text {arity }\left(R_{i}\right)} \times T_{I}^{\prime}$, where each $\mathbf{R}_{\mathrm{i}}^{\prime}$ is finite.

For instance, assume a database schema $\rho$ consisting of a single binary predicate symbol work, storing for each employee the department in which she is employed. The TDB schema $\rho^{\prime}$ consists of the relation work', called a validtime stale lable, which adds a temporal argument to the original relation, called the validtime of the table. 'The temporal argument can be used to store the history (and perhaps oven future plans) of departments in which employees are employed. Following a suggestion of $(\Lambda n-$ droutsopoulos, 1996), we also include rolations mapping names of calendrical items to temporal intervals in $\rho^{\prime}$. For instance, we store a relation year' mapping the year 2000 to the interval [1.1.2000-31.12.2000] (which in turn is mapped to an element of $\left.T_{1}\right)$.

We now describe the translation process.

\section{The translation process}

The translation process accepts input NL questions in a controlled subset of NI. Restricting input language in this way enables eflective processing of a sufficiently rich fragment while avoiding many of the well-know problems of unrestricted NI. We use a formal grammar in the TLG framework. Our grammar is specially designed for use with a particular 'I'DB schema. Future work will allow easier configuration of the grammar with respect to the schema.

Our grammar is based on work on an independently motivated theory of the semanties of temporality. Most of the research in this ficld (sce (Stcedman, 1997) for a survey) has focused on the issues of tense and aspect. We handle tense, but purposefully not aspect, which plays 
an important role in (Androutsopoulos, 1996). Aspect, which is used to reflect speakers' temporal viewpoint with respect to reported situations is an important facet of NL temporality. Ilowever, its relevance to TDBs is questionable, as it is unlikely that a realistic TDDB would actually encode such subjective viewpoints. Morcover, handling aspect requires postulating a more complex data model. For instance, (Androutsopoulos, 1996) augments the TJDB model with cvent-like "occurrence identifiers", and adds an additional argument to temporal relations indicating whether a given event has culminated or not. While such devices may perhaps be linguistically justified, it is unclear whether the TDB community would adopt such augmentations of the model.

Instead, following (Pratt and Francez, 2000; Nelken and Francez, 1999) our focus is on sentences modified by temporal PPs. These PPs are analyzed as variants of standard generalized quantifiers (Barwisc and Cooper, 1981), in which quantification is over time. Using this framework, we handle questions that refer explicitly to the temporal dimension (c.g. When/during which year ...) as well as questions in which temporality is implied by the TDB context (e.g. Did Mary work in marketing?, Which employees worked in marketing?). We handle both clausal and phrasal temporal l.P's (e.g. after John worked in R\&L, during every year). An important strength of this semantic theory is that it allows for arbitrary iteration of PPs (e.g. one month during every year until 1992). In addition, our grammar also handles quantification over individuals (c.g. some employee), coordination and negation.

Input questions are parsed using a lexicalized type-logical grammar. Lexical items are associated with a syntactic category and a higherorder lambda-term representing its scmantics. Taking advantage of 'I'LG's elegantly tight coupling of syntax and semantics, parsing and construction of a semantic representation in the form of an $\mathrm{I}_{\text {Allen }}$ formula proceed simultaneously, in a bottom-up fashion. We have found using TLG to be advantageous over a featurestructure based formalism (such as HPSG as in (Androutsopoulos, 1996)), since formula construction is an integral part of the parsing and does not require complex ad-hoc manipulations of feature structures.

Using a particular grammar helps reduce some of the ambiguity inherent in unrestricted NL. lor instance, whereas in general a preposition such as at is ambiguous between a temporal and a locative interpretation, the choice of the complement NP relative to a given schemainduced grammar deterministically fixes the interpretation. As another example, whercas itcrating sevoral temporal PPs (e.g. during some month every year) opens up exponcntial scoping possibilities, some choices are eliminated by world knowledge, which is encoded in the grammar (c.g. every year must have higher scope than some month since months are included in years and not vice-versa). In cases of remaining ambiguity, the user is presented with all the distinct possibilitios. Future work will allow the user to make informed choices between different possible readings, e.g. by presenting him with NI, paraphrases of the alternatives.

We translate NI, questions into $\mathrm{I}_{\text {Allen }}$. The main reason for not translating directly to $\mathrm{SQI} /$ Temporal is that the latter is not closed for sub-formulac, i.c. a sub-formula of a wellformed query is not necessarily well-formed. Since $\mathrm{L}_{\text {Allen }}$ is closed for sub-formulae, compositionally constructing formulac while parsing in a bottom-up fashion becomes much casier.

$\mathrm{L}_{\mathrm{All}}$ is defined as follows (Toman, 1996). l et $\rho$ be the database schema $\left(R_{1}, \ldots, R_{k}\right)$. Let:

$$
L::=R_{i}(\mathrm{x}, I)|L \wedge L| \neg L|\exists x . L| \exists I . L|x=y| I \sigma J
$$

where $x, y$ are variables over $D, \mathrm{x}$ is a vector of such variables, $I, J$ are variables or constants over $T_{I}$, and $\sigma$ is one of the operators: precedes, meets, overlaps, equals, contains. $\mathrm{L}_{\mathrm{Allen}}$ is defined as the set of formulae $\varphi \in I$ that contain at most one free variable over 'T'. The answer to a formula $\varphi$ relative to a TDB $\mathcal{D}$ is $\{\mathrm{x}, I \mid D \models \varphi(\mathrm{x}, I)\}$.

To illustrate, consider the NL, question: During which years did Mary work in marketing? The $\mathrm{I}_{\text {Allen }}$ representation for it, is constructed in a bottom-up manner. The meaning representation of the main clause Mary worked in marketing is constructed as:

$\lambda I . \exists J($ work (mary, marketing,$J) \wedge J \subseteq$ past $\wedge J \subseteq I)$

In this formula, $I$ denotes a Reichenbachianlike reference time, $J$ denotes a time interval 
during which Mary worked in marketing, which is located in the past (the contribution of the tense) and is included within $I$.

The meaning of the full question is constructed by applying the meaning of the interrogative temporal P'P during which year to the meaning of the clause. Without going into details, the result is:

$\operatorname{yoar}(I) \wedge \exists J($ work (mary, marketing, $J)$ $\wedge J \subseteq$ past $\wedge J \subseteq 1)$

The eflect of applying the Pl' is that the variable $I$ is now both free and restricted to be the time of a year. 'The answer to the formula is the set of intervals $I$ that are years, and during which there is an interval I contained in the past, during which Mary worked in marketing. We allow iterated Pl's to apply in a similar manner.

Not every $L_{\Lambda \mathrm{ll} \text { en }}$ formula corresponds to an evaluable SQI/Temporal query. In particular, formulae might have an infinite answer. l'ormulae that are sale from this and related problems are termed domain-independent (Gekler and 'Topor, 1991; Abiteboul of al., 1995). Domain independence is an undecidable semantic property. llowever, we impose certain syntactic restrictions on generated formulae that ensure it. 'These restrictions also simplify the translation task from Lallen to SQJ /Temporal. 'I'lis translation is based on a modifieation of the translator from first-order Tl, over time-pointis to N'TSQL of (Bochlen ot al., 1996).

T'he syntactically restricted version of L N Nen we use has the unicue advantage of being very close both to the language used in (Nelken and Fances, 1999) on the one hand and to SQJ/T'temporal on the other. The semanties of NI temporal expressions is often expressed using explicit reference to intervals. Likewise, SQL/Temporal has Allen-style operators over intervals. Androutsopoulos (1996) uses a customized TT, as an intermediate language, in which temporal relations are cncoded using temporal operators rather than explicit refercnce to intervals. We have found using a syntactically restricted version of $J_{\text {Allen }}$ to be advantageous, as it actually simplifics tho translation.

Continuing our previous example, the result ing $\mathrm{L}_{\text {Allen }}$ formula is subsecpuently translated into the following SQL/Temporal cuery:

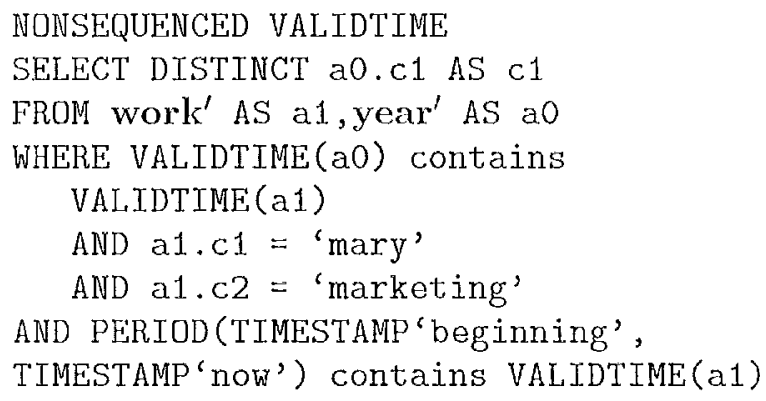

The query asks for the first argument of the relation instance year' such that the relation instance work' includes a tuple consisting of 'Mary', 'marketing' and a valid timo, which is temporally included in the valid time of the year, as well as in the interval starting at the 'beginning' of time and ending 'now' - vi\%. the past. 'The 'l']ls responds by returning a table containing exactly the requested year names.

\section{Conclusion}

The addition of the temporal dimension to datablyase systems incroases their power but also their complexity. To increase the usability of T'IJBs, we present a prototype controlled NL interface to a TI) B. Our semantic focus is on the use of temporal generalized quantifiers, based on (Pratt, and lrances, 2000), rather than tense and aspect. As argued by (Copestalie and Jones, 1990), hanelling quantification is one of the areas in which $\mathrm{Nl}$, interfaces have a potential advantage over both formal languages and graphical user interfaces.

In comparison with previous work, we are able to considerably simplify the transtation mothod. lirst, using 'TLG, rather than a feature-structure formalism provides a much simpler method for constructing semantic representations. Second, using $L_{\Delta \| l n}$ as an intermediate meaning representation language yiokds a much more straight forward translation than using a restricted 'I'l.

One must bear in mind, that our implementation is at the prototype stage. Turning it into a practical tool would require considerable work, as is true of most comparable systems. Future work includes increased $\mathrm{Nl}$, coverage, adding a disambiguation module, handling nominal and temporal anaphora, allowing multiple-sentence (pueries, and generation of $\mathrm{NI}$, answers from the results presented by the Tl])B. 


\section{References}

S. Abiteboul, R. Hull, and V. Vianu. 1995. Foundations of Dalabases. Addison-Wesley.

J .F. Allen. 1983. Maintaining knowledge about temporal intervals. CACM, 26(11):832-843, nov.

I. Androutsopoulos, G. D. Ritchie, and P. Thanisch. 1995. Natural language interfaces to databases - an introduction. Natural language Engineering, 1(1):29-81.

I. Androutsopoulos. 1996. A principled Framework for Constructing Natural Language Interfaces to Temporal Databases. Ph.D. thesis, University of Edinburgh.

J. Barwise and R. Cooper. 1981. Generalized quantifiers and natural language. Linguistics and Philosophy, 4:159-219.

Michacl H. Bochlen, Jan Chomicki, Richard T. Snodgrass, and David Toman. 1996. Querying TSQL2 databases with temporal logic. In Proceedings of the 5th International Conference on Extending Database Technology (EDBT), Avignon, France.

M. H. Boehlen. 1995. Temporal database system implementations. Unpublished manuscript, Department of Mathematics and Computer Science, Aalborg University.

B. Carpenter. 1998. Lectures on Type-Logical Semantics. MIT Press.

B. Carpenter. 1999. Type-logical grammar theorem prover. hittp://www.colloquial.com/tig.

J. Clifford. 1990. Formal Semantics and Pragmatics for Natural Language Querying. Cambridge University Press, Cambridge. Cambridge Tracts in Theoretical Computer Science 8.

A. Copestake and K. Sparck Jones. 1990. Natural language interfaces to databases. The Knowledge Engineering Review, 5(4):225249.

R. S. Crouch and S. G. Pulman. 1993. Time and modality in a natural language interface to a planning system. Artificial Intelligence 63, 63:265-304.

A. Van Gelder and R.W. Topor. 1991. Safety and translation of relational calculus queries. ACM Transaction on Database Systems, 1.6(2):235-278, June.

E. W. Hin richs. 1988. Tense, quantifiers, and contexts. Computational Linguistics, 14:3-
14.

G. Morrill. 1998. Type Logical Grammar: Categorial Logic of Signs. Kluwer, Dordrecht.

R. Nelken and N. Francez. 1999. A semantics for temporal questions. In Geert-Jan M. Kruijf and Richard T. Ochrle, editors, Proceedings of Formal Grammar 1999, pages 131-142.

C. R. Perrault and B. J. Grosz. 1988. Natural language interfaces. In H. E. Shrobe, editor, Exploring Artificial Intelligence, pages 133172. Morgan Kaufmann Publishers Inc., San Mateo, California.

C. Pollard and I. A. Sag. 1994. ITead Driven Phrase Structure Grammar. University of Chicago Press, Chicago.

I. Pratt and N. Francez. 1997. On the semantics of temporal prepositions and preposition phrases. Technical Report UMCS-97-42, University of Manchester, Department of Computer Science.

I. Pratt and N. Francez. 2000. Temporal prepositions and temporal generalized quantifiers. To appear in Jinguistics and Philosophy.

R. T. Snodgrass, editor. 1995. The TSQL2 Temporal query language. Kluwer, Norwell. MA.

R. T. Snodgrass. 2000. Developing TimeOriented Database Applications in SQL. Morgan Kaufmann Publishers, San Francisco, CA.

M. Steedman. 1997. Temporality. In J. Van Benthem and A. 'Ter Meulen, editors, Handbook of logic and language, pages 895-938. Elsevier.

A. Steincr. 1997. A Generalization Approach to Temporal Data Models and their Implementation. Ph.D. thesis, Department Informatik, ETII Zurich.

A. Tansel, J. Clifford, S. Gadia, S. Jajodia, A. Segev, and R. Snodgrass (cds.). 1993. Temporal Databases: Theory, Design, and Implementation. Database Systems and Applications Scries. Benjamin/Cummings, Redwood City, CA.

D. 'Ioman. 1996. Point vs. Interval-based Query Languages for Temporal Databases. In Proceedings of the ACM SIGACT-SIGMODSIGART PODS, pages 58-67, Montreal, Canada, June. 